\title{
Gaze Following and Joint Attention in Rhesus Monkeys (Macaca mulatta)
}

\author{
Nathan J. Emery, Erika N. Lorincz, David I. Perrett, Michael W. Oram, and Christopher I. Baker \\ University of St. Andrews
}

\begin{abstract}
Gaze and attention direction provide important sources of social information for primates. Behavioral studies show that chimpanzees spontaneously follow human gaze direction. By contrast, non-ape species such as macaques fail to follow gaze cues. The authors investigated the reactions of rhesus macaques (Macaca mulatta) to attention cues of conspecifics. Two subjects were presented with videotaped images of a stimulus monkey with its attention directed to 1 of 2 identical objects. Analysis of eye movements revealed that both subjects inspected the target (object or position attended by the stimulus monkey) more often than the distractor (nonattended object or position). These results provide evidence that rhesus monkeys follow gaze and use the attention cues of other monkeys to orient their own attention to objects.
\end{abstract}

The ability to gaze follow has been demonstrated most successfully in human infants. The age at which an infant first follows another's gaze is controversial, ranging from 6 to 18 months of age (Butterworth \& Cochran, 1980; Butterworth \& Jarrett, 1991; Corkum \& Moore, 1994; Scaife \& Bruner, 1975). These age differences may be due to differences in methods (variation in angle of gaze; the use of an experimenter vs. the infant's mother) or in the definitions. Investigators use the terms joint attention and gaze following interchangeably. Scaife and Bruner (1975) defined the ability to gaze follow as the "visual attention of the mother-infant pair ... directed jointly to objects and events in the visual surround" (p. 265), yet in their study there was no specific focus of attention, such as an object. By contrast, other researchers do not stress the object of attention in definition (e.g., "the ability to follow another's direction of gaze," Corkum \& Moore, 1994, p. 62; "looking where someone else is looking," Butterworth, 1991, p. 223) yet may nonetheless use an object of attention during study (Butterworth \& Cochran, 1980; Butterworth \& Jarrett, 1991).

Nathan J. Emery, Erika N. Lorincz, David I. Perrett, Michael W. Oram, and Christopher I. Baker, School of Psychology, University of St. Andrews, Scotland.

Preliminary results of this study were presented in March 1996 at the Third Cognitive Neuroscience Society Meeting, San Francisco. This study is supported by grants from the U.K. Medical Research Council, the Biotechnology and Biological Sciences Research Council (U.K.), a Fyssen Foundation postdoctoral fellowship (France), and St. Leonard's College, University of St. Andrews.

We thank Brian Kirk, Hilda Dickie, Wendy Taylor, and Heather Bowman for technical assistance. We also thank Andy Whiten and Daniel Povinelli for comments on an earlier version of the manuscript.

Correspondence concerning this article should be addressed to Nathan J. Emery, David I. Perrett, or Erika N. Lorincz, School of Psychology, University of St. Andrews, St. Andrews, Fife KY16 9JU, Scotland. Electronic mail may be sent via Internet to nje1@st-andrews.ac.uk,dp@st-andrews.ac.uk, or ell@st-andrews.ac.uk, respectively.
We would like to suggest that gaze following and joint attention are different yet intimately related abilities (probably with different developmental and phylogenetic time courses). Gaze following could be defined as the ability of one individual $(X)$ to follow the direction of gaze of a second individual $(\mathrm{Y})$ to a position in space (not an object, see Figure 1a). Joint attention has the additional requirement that $X$ follows the direction of Y's gaze to the object (Z) that is the focus of Y's attention (see Figure 1b). Joint attention thus requires extra computation to process the object of attention, not just the direction of gaze.

A large body of evidence suggests that feral primates can follow gaze. Monkeys and apes can gain information about available food sources, social dominance, and the location of predators from the attention direction of conspecifics (Chance 1967; Menzel \& Halperin, 1975; van Schaik, van Noordwijk, Warsono, \& Sutriono, 1983; Whiten \& Byrne, 1988). If an object is sufficiently interesting to capture one individual's attention, then this directed interest may draw another individual's attention to the same object. In field studies, however, individuals may come to fixate the same object because of the object's inherent interest even if they do not follow gaze.

In some primate groups, the level of attention afforded to an individual has been suggested as indicating the animal's dominance in a social hierarchy (Chance, 1967). Determining which individual receives the largest amount of attention would be impossible without gaze following and joint attention. Whiten and Byrne (1988) suggested that visual attention is an important component to what they termed tactical deception. An example of this would be, $X$ sees a patch of fruit to his right but looks to the left, so that $Y$ will look in the opposite direction to the fruit. This might enable $\mathrm{X}$ to approach and take the fruit while $\mathrm{Y}$ 's attention is elsewhere.

Gaze direction in non-ape species is used as a signal in agonistic encounters. Direct gaze is a threatening gesture (Redican, 1975), and averting gaze is used to diffuse the meaning of such a gesture (Altmann, 1967). Gaze following 
(a)

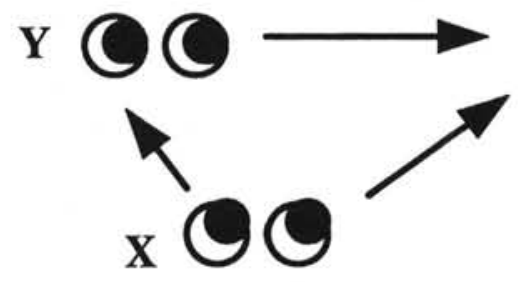

(b)

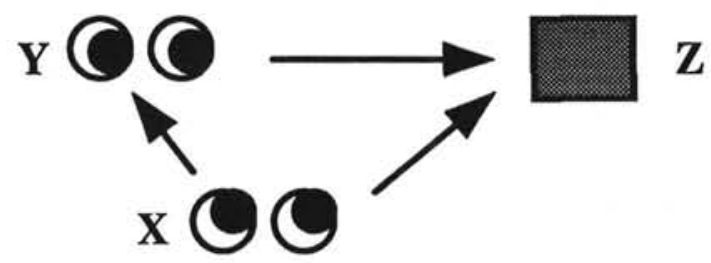

Figure 1. Levels of social interaction using gaze: (a) schematic representation of gaze following (dyadic relationship) in which an observer $(\mathrm{X})$ follows the direction of gaze of the observed individual (Y) and (b) joint attention behavior (triadic relationship) in which an observer (X) follows the gaze of the observed (Y) onto an object (Z).

is important in both instances. Individual $\mathrm{Y}$ must recognize that Individual $\mathrm{X}$ is threatening him and not another animal, and $\mathrm{X}$ needs to recognize that $\mathrm{Y}$ is looking elsewhere and has responded subordinately to the threat to terminate an unpleasant situation.

A primate may learn something about an object by observing another individual physically interacting with that object. This is the basis for forms of social learning such as local (or stimulus) enhancement, imitation (Whiten \& Ham, 1992), and fear conditioning (Mineka, Davidson, Cook, \& Keir, 1984). Physical interaction between agent and object may not be required; gazing alone may be a salient enough cue for social learning about objects.

Laboratory studies of gaze processing have concentrated almost exclusively on human infants and great apes. Povinelli and Eddy $(1994,1996 a, 1996 \mathrm{~b})$ investigated whether young chimpanzees (Pan troglodytes) would follow an experimenter's gaze (head and eyes or eyes alone) to one side rather than the opposite. The subjects correctly oriented to the attended side and also followed the experimenter's gaze when directing their attention to objects outside the chimpanzee's view, such as behind them (Povinelli \& Eddy, 1996a). Povinelli and Eddy (1996a) suggested that spontaneous gaze following (as an orienting reflex) may be present in monkeys and probably other social animals.

Itakura (1996) recently studied 11 species of prosimians, monkeys, and apes in their ability to follow a human experimenter's gaze (eyes, head, and manual pointing in the same direction). Only orangutan and chimpanzee subjects made greater than $70 \%$ correct responses. The non-ape subjects (brown lemur, black lemur, squirrel monkey, brown capuchin, white-face capuchin, stump-tailed macaque, rhesus macaque, pig-tailed macaque, and tonkean macaque) did not respond above chance.

This result is interesting in the light of neurophysiological studies, recording from single neurons in the rhesus macaque temporal cortex (Perrett, Hietanen, Oram, \& Benson, 1992; Perrett \& Mistlin, 1990; Perrett et al., 1985) and amygdala (Brothers \& Ring, 1993; Leonard, Rolls, Wilson, \& Baylis, 1985). Cells selectively responsive to particular views of human or monkey heads (front, back, profile, up, and down) were discovered in the early 1980s (Bruce, Desimone, \& Gross, 1981; Perrett, Rolls, \& Caan, 1982). Cells in the superior temporal sulcus and amygdala are also sensitive to the direction of eye gaze (Brothers \& Ring, 1993; Perrett et al., 1985, 1992). Perrett et al. (1992) suggested that one function of such cells would be to assist in the recognition of where another individual is looking. The monkey would appear to have brain systems dedicated to processing where others are attending and how others are interacting, both with inanimate objects and more socially with conspecifics (Brothers, 1990; Perrett \& Emery, 1994). This view of a dedicated neural system would presume that rhesus monkeys, and probably other primates, possess the behavioral capacity to follow gaze. Such behavior may not have been shown previously because the experimental paradigms used so far have not asked specific questions of gaze following. Instead the studies have focused on understanding the mental significance of gaze.

In an attempt to resolve the differences between the physiological evidence of macaque brain mechanisms processing gaze cues and the lack of behavioral demonstration of gaze following in captive macaques, we constructed an experimental paradigm that would not require extensive training and that would specifically record the responses of monkey subjects viewing other individual's gaze behavior. This paradigm used conspecific stimulus monkeys to provide attention direction cues and measured the spontaneous eye movements of test subjects, rather than requiring the subjects to provide an action for reward.

\section{Method}

\section{Subjects}

The subjects were 2 male rhesus macaques, Steve (age 4 years), and Terry (age 3 years), who were also subjects in ongoing neurophysiological studies that required stable eye movement recording (see Perrett et al., 1985, for details of neurophysiological procedures). The subjects had previously been shown a video film of conspecific monkeys and other animals. They had also been exposed to slides and video disk images of humans, monkeys, and other animals. The subjects were born and reared in a social colony of mesus monkeys. During the period of the experiment, the subjects were housed individually, but remained in auditory and visual contact with the other monkeys. The subjects were familiarized with the test room and a primate chair with head restraint. During testing, fruit juice was available ad libitum. All experiments were performed under a U.K. Home Office Project and Personal License, and all experiments were regulated by the University of St. Andrews (Scotland) Animal Code of Practice. 


\section{Test Stimuli}

Test stimuli were created by videotaping (Panasonic SVHS video camera) a stimulus monkey sitting at a square window (width $=26.5 \mathrm{~cm}$ ) with the area surrounding the window blacked out. The stimulus monkey was filmed maintaining attention in one direction (down left [DL], down right [DR], up left [UL], or up right [UR]). The stimulus monkey was attracted to look intently in one direction by presenting interesting stimuli at the desired locations. A JVC color monitor was set up on a stand in one of the four desired attention locations (DL, DR, UL, or UR). From the stimulus monkey's perspective, the left and right positions of the monitor were separated by $120^{\circ}$. Various images were presented over the monitor, played on an Akai VCR, including videotaped images of various animals in a zoo, cartoons, and pictures of monkeys. Particular hand puppets, face masks, and toys were also presented from behind an occluder to achieve the same purpose. These methods were sufficient to allow video recording of 20 segments of film (5 each of DL, DR, UL, and UR), which would be converted to test trials. These segments were edited using a Panasonic SVHS VCR (NV-FS200B) and a Panasonic VHS video mixer (WJAVE7) to blank off the area on either side of the window, leaving only the centrally positioned stimulus monkey visible.

Two objects were added to each trial segment. The objects were identical mirror images of each other and were recorded onto videotape at the same time and at opposite lateral corners of the screen. This was achieved using a Fairlight CVI (computer-video effects machine). One object was filmed using a Panasonic SVHS video camera (F10CCD) at either the bottom left or top left of the screen and mirrored about the center of the screen (Fairlight CVI). This provided the illusion that two identical objects were entering the screen at the same time and at the same mirrored spatial location, for example, one object enters bottom left, whereas the other enters bottom right. A different object was used for each trial. The objects were all novel to the subject, all approximately the same size and made small movements up and down.

The specific object or position that the stimulus monkey was attending to is referred to as the target object or attended target position (T), and the object at the opposite position is referred to as the distractor object or nonattended distractor position (D). The contents of the central window are referred to as the stimulus monkey (M), and everywhere else on the screen is referred to as elsewhere (E). An idealized example of a frame from the test tape is presented in Figure 2. The projected distance between target and distractor on the actual test tape was $40^{\circ}$ (physical distance $=150$ $\mathrm{cm}$ ), and the physical distance between the stimulus monkey's head and the target was on average $90 \mathrm{~cm}$ (minimum $=80 \mathrm{~cm}$, maximum $=100 \mathrm{~cm}$ ). During some trials the stimulus monkey's head moved toward and away from the target. Measurement showed that on average across trials the stimulus monkey's head was marginally closer to the distractor than to the target. In all trials the orientation of the stimulus monkey's gaze was identical to the orientation of the head.

Two final test tapes were prepared. Condition 1 included 20 trials with target and distractors down left and down right. Condition 2 included 20 trials with target and distractors up left and up right. The left/right positions of the target and distractor were randomized across trials.

\section{Experimental Procedure}

The subject was seated $4 \mathrm{~m}$ from the projector screen, at the same height as the projected stimulus monkey. An infrared camera and a half-silvered mirror were attached to the front of the primate

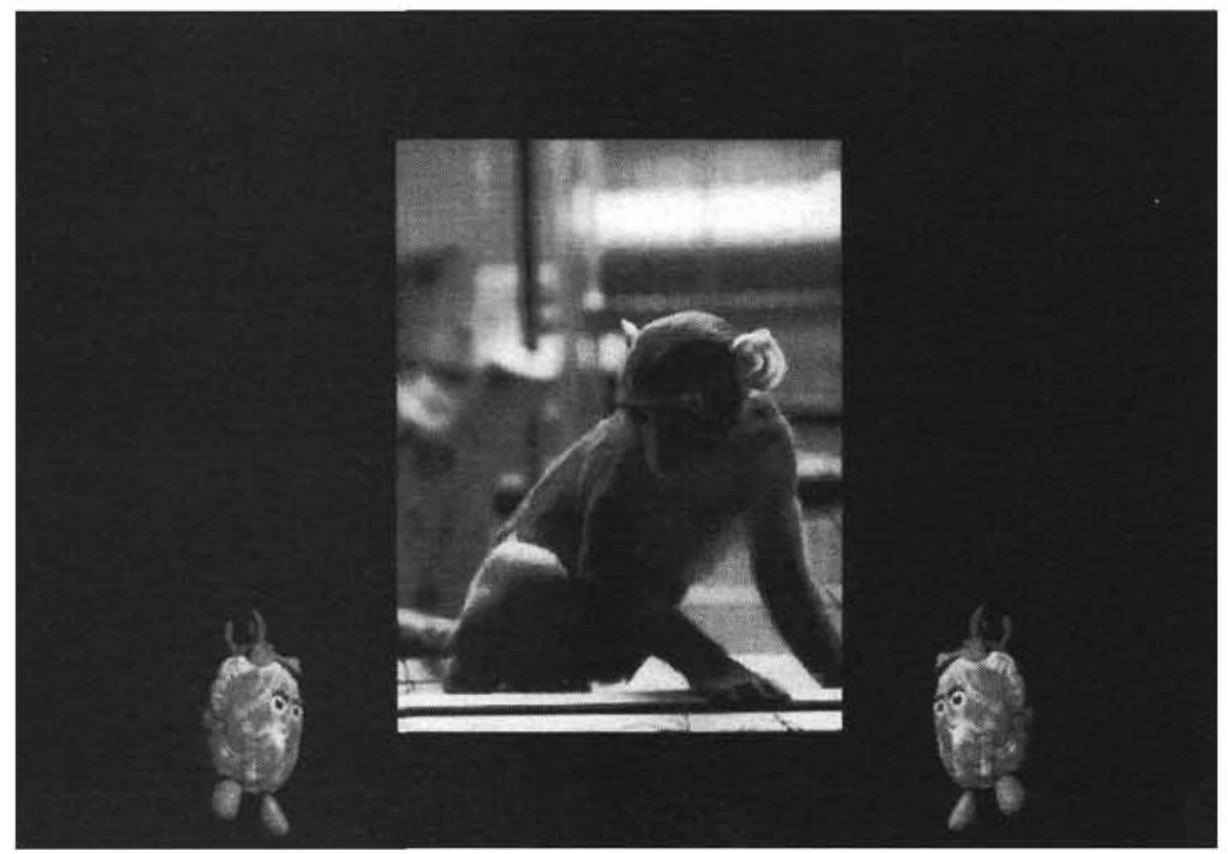

Figure 2. Spatial representation of test stimuli. The stimulus monkey is positioned relatively central attending down left to the target object and away from an identical distractor object (Condition 1, monkey-and-objects time period). During testing, the subject's eye movements and a frame/time code are mixed onto a copy of this test tape (not shown). For test stimuli eyes, head and body of the stimulus monkey had the same direction of orientation. 
chair. This arrangement allowed the subject's eye movements to be videotaped while viewing test stimuli through the mirror (see Figure 3). A VITC time-code generator and frame counter (Horita VG50) allowed the addition of a time and frame code to the bottom of the eye movement recording tape. The video stimuli were projected in a darkened room to avoid any visual distraction for the subject. The test tape was presented on a VCR (Panasonic NV-FS200B) with an output to a Sony color video projector (VPH-1041 QM). The output from the eye movement camera was video-mixed (Panasonic VHS video mixer, WJAVE7) onto a copy of the test stimuli tape with the frame code and recorded onto VHS tape on a VCR (Panasonic NV-FS200B).

Three time periods were present during each trial. A 1.0-s tone preceded the beginning of the trial in which the stimulus monkey appears (monkey-only condition). After 2-3 s, the objects appear (monkey-and-objects condition). The stimulus monkey and objects remained on screen for 7-9 s, after which the stimulus monkey disappeared off screen. The objects remained on screen for a further $2-3 \mathrm{~s}$ (objects-only condition). There was an intertrial period of 5-6 $s$ before the next tone and trial. Trials varied in duration because of changes in the length of time that the stimulus monkey's attention was captured in the test direction during the original filming of the test tapes.

\section{Scoring Gaze Direction}

For each trial, the frame counts ( 1 frame $=40 \mathrm{~ms}$ ) of the critical events (trial begins, targets appear, stimulus monkey disappears, and trial ends) and the stimulus monkey's orientation were recorded onto score sheets by one observer by projecting the test video with the stimulus monkey, objects, and eye movements present.

Analysis of the location of inspections was performed by two observers who were unaware of the orientation of the stimulus monkey. This analysis was achieved by blanking off the region of the test tape containing the stimulus monkey with the video mixer. The resulting image contained the subject's eyes and the objects when they appeared. Analysis was performed on the test video (minus the central region) projected onto the screen so that the distance between the eyes in the projected image of the subject's face was $18 \mathrm{~cm}$ (i.e., $32 \mathrm{~cm}$ between pupils).
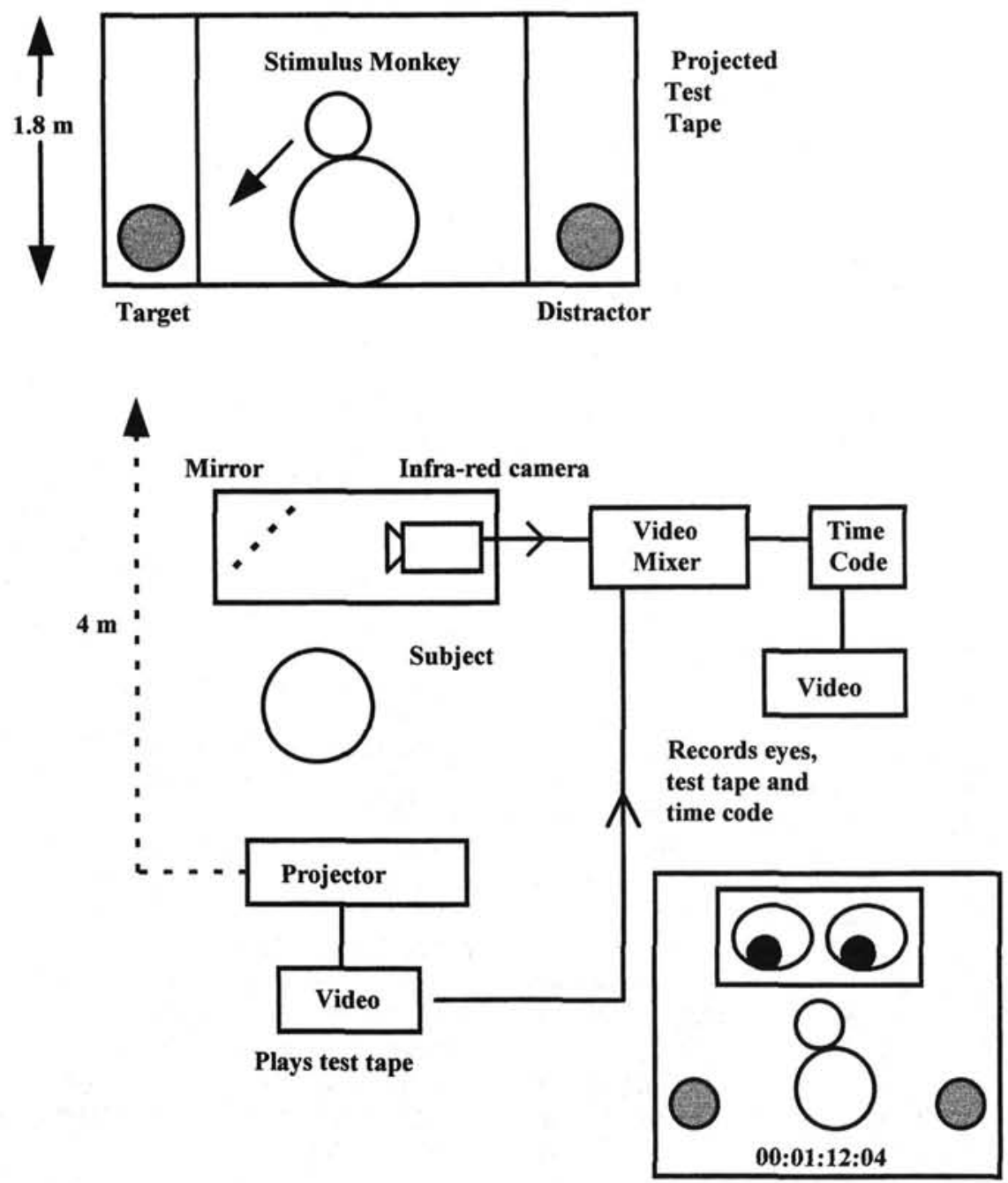

Figure 3. Recording and stimulus presentation setup. The test video was presented on a large white screen $4 \mathrm{~m}$ from the subject. The subject's eye movements were recorded by an infrared camera positioned in front of the subject. The eye movements were then mixed onto a copy of the test tape for off-line analysis. A frame/time code is also added to this copy of the test tape. 
Attribution judgments were made by scorers for each of the subject's inspections. A fixation was defined as the subject's eyes remaining static for at least two to three frames duration (80-120 $\mathrm{ms}$ ). Multiple successive fixations on different regions of the same object without intervening fixations of other objects or positions were scored as a single inspection. During a saccade the subject was scored as not looking at anything. Inspections were attributed to one of four areas: (a) left target, (b) right target, (c) center (stimulus monkey), and (d) elsewhere. The position of each inspection and its frame count were recorded for analysis.

An off-line analysis, which combined blind scoring of inspections (by one rater) and records of stimulus monkey orientation, reclassified the four inspection positions to inspection of target, distractor, stimulus monkey, and elsewhere. Interobserver reliability was calculated for a sample of 20 trials by correlating the number of inspections attributed to different positions per trial by two scorers analyzing videos independently. The independent blind scoring correlated highly for the different positions: stimulus monkey, Pearson's $r^{2}(19)=.59, p<.01$; target, $r^{2}(19)=.69, p<$ $.01 ;$ and distractor, $r^{2}(19)=.76, p<.01$.

\section{Data Analysis}

From the score sheets, the mean number of inspections made of each of the four positions were calculated for each trial. The duration of each inspection was also calculated from the frame count. The number of inspections and duration per inspection were compared for the whole trial using a three-way analysis of variance (ANOVA) in which the variables were subject (Steve, Terry), condition (down/up), and position (T, D, M, and E), and NewmanKeuls post hoc tests were used to determine specific significant differences. The number of inspections at each position $(T, D, M$, and E) was also measured for each individual time period. For each time period, the percentage of inspections of the target plus distractor positions were compared using a binomial test, with data combined for each subject.

\section{Results}

Both subjects' eye movements were recorded for Condition 1 (down) and Condition 2 (up) for 20 trials each. The results were analyzed for the whole trial using a three-way ANOVA (subjects, condition [down/up], and inspection position as main variables). There was a significant main effect of position on the number of inspections made, $F(3,3)=16.05, p<.05$. Of greatest importance, the number of inspections on the target position was significantly greater than the number of inspections on the distractor position (Newman-Keuls, $p<.05$ ). Figure 4 illustrates that although Steve made more inspections than Terry-main effect of subjects, $F(1,76)=81.46, p<$ .001 - the pattern of inspection across different positions was constant across the 2 subjects-no interaction between subjects and position, $F(3,228)=1.32, p=.27$.

Of less importance, there was no significant main effect of condition, $F(1,1)=33.28, p=.11$, as the number of inspections was not different between the up and down conditions. Other interactions between the main variables were all nonsignificant: subjects and condition, $F(1,76)=$ $0.18, p=.68$; condition and position, $F(3,3)=1.55, p=$ .36 ; and subjects, condition, and position $F(3,228)=1.0$, $p=.39$.

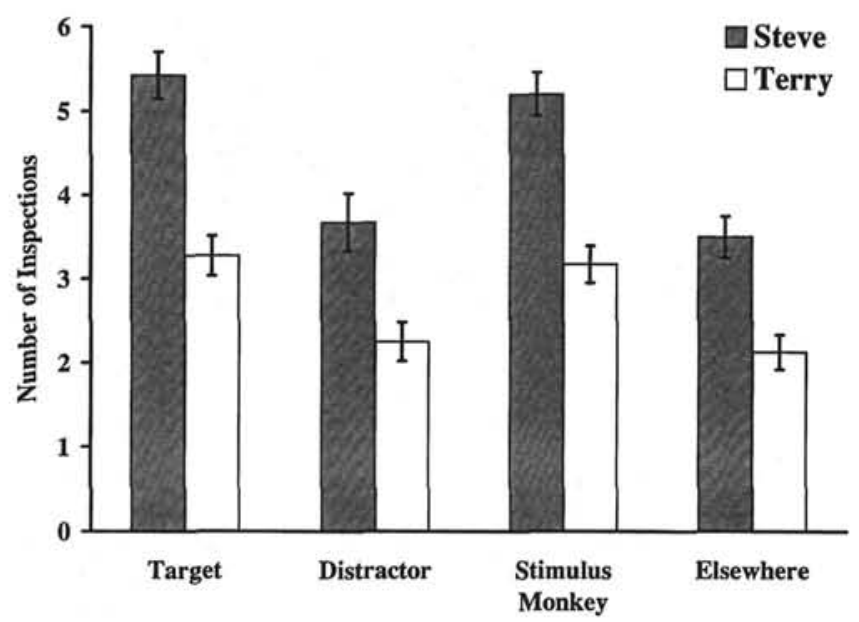

Figure 4. Pattern of inspections within test stimuli. Mean number of inspections ( $\pm S E M$ ) made to each position (target, distractor, stimulus monkey, and elsewhere) for the whole trial. Data were averaged for the 40 trials (in Conditions 1 and 2) separately for each of the 2 subjects. Note that the pattern of inspections across positions is the same for both subjects even though Steve made more inspections overall compared with Terry.

Behavior during the individual time periods was analyzed using a binomial test. The number of inspections made by each subject of the target position (T) or the distractor position (D) for each time period for the total 40 trials was calculated as a percentage of the total number of inspections of the target position plus the distractor position $(T+D)$. For the monkey-only time period, the proportion of inspections of the target position was significantly greater than that for the distractor position (binomial test, $Z=4.7, p<.01$ ). During the monkey-and-objects time period, the number of inspections of the target was also significantly greater than that for the distractor (binomial test, $Z=2.6, p<.05$ ). There was no significant difference between the proportion of inspections of the target and distractor for the final objectsonly time period (binomial test, $Z=0.9, p=.18$; see Figure 5 for these results).

The number of frames at each of the four positions for the two subjects and conditions were analyzed using a threeway ANOVA. There was no significant main effect of position, $F(3,3)=3.6, p=.16$, and therefore no difference between the number of frames spent looking at the target and the distractor (see Figure 6). There was no significant main effect of subjects, $F(1,76)=1.52, p=.22$, or condition, $F(1,1)=2.41, p=.36$, and no interaction between subjects and condition, $F(1,76)=0.51, p=.48$, or between condition and position, $F(3,3)=0.94, p=.52$. There was a significant interaction between the subjects and position, $F(3,3)=5.5, p<.001$. Steve spent significantly more time looking at the target than the distractor (Newman-Keuls, $p<.05$ ), whereas the difference for Terry was nonsignificant.

The duration of inspections over the whole trial was further analyzed by using comparable methods to those above, with time spent inspecting the target and distractor expressed as a proportion of total time spent inspecting both 

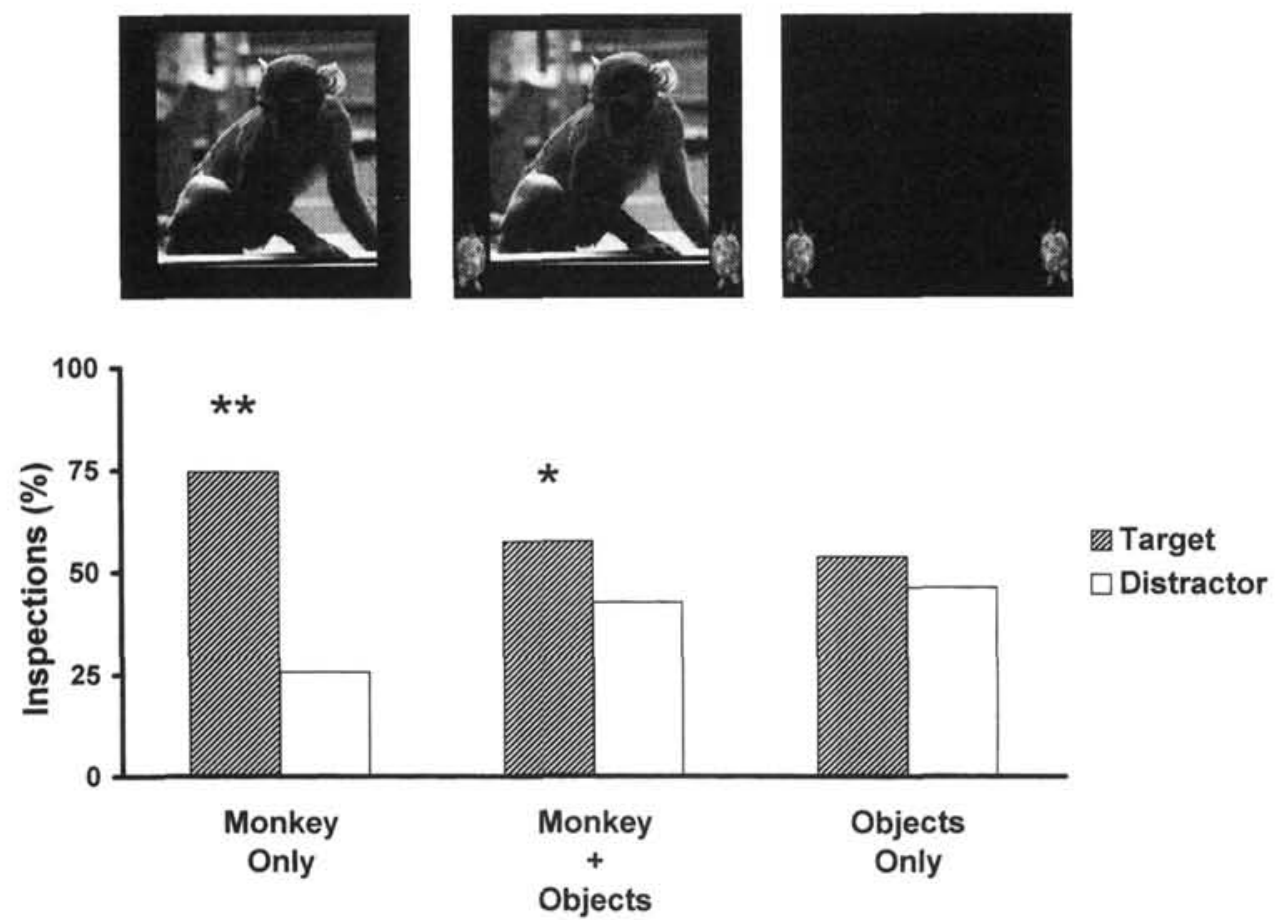

Figure 5. Proportion of target and distractor inspections across different trial periods. Upper: stimuli during the successive periods of the trial (monkey only, monkey + objects, objects only). Lower: mean proportion of inspections made to the target $(T)$ and distractor (D) positions (expressed as a percentage of $\mathrm{T}+\mathrm{D}$ ) for each trial period. ${ }^{*} p<.05 .{ }^{* *} p<.01$.

the target and distractor for each of 40 trials. The average of both subjects indicated that subjects spent a greater proportion of time inspecting the target position than the distractor position (binomial test, $Z=12.2, p<.01$ ).

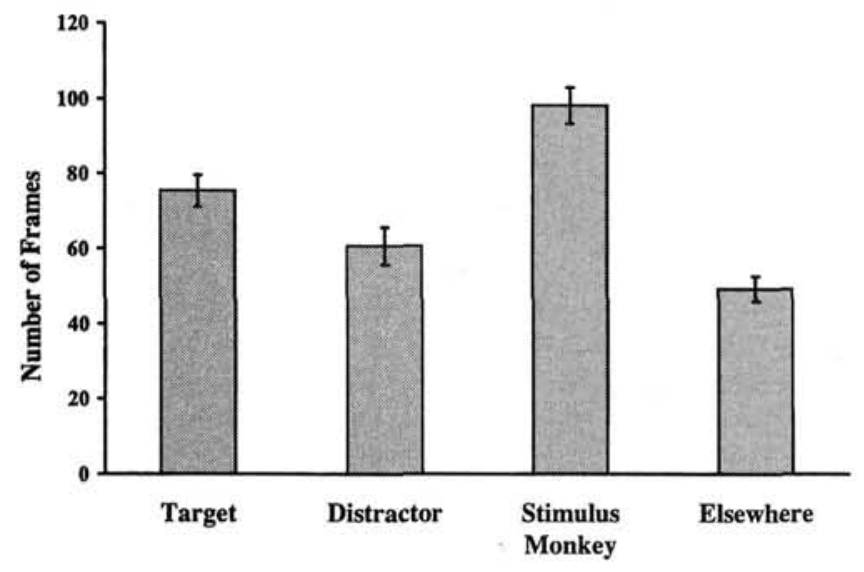

Figure 6. Duration of inspections. The average number of frames ( $\pm S E M$ ) spent inspecting the different stimulus positions for the whole trial. Data for the 40 trials for each of the two subjects in Conditions 1 and 2 have been averaged.

\section{Discussion}

The results of this study provide the first experimental evidence that rhesus monkeys follow another monkey's gaze and use their gaze cues to orient their own attention to a specific object. The subjects followed the stimulus monkey's line of sight before any objects were presented and inspected the attended target object more than the identical nondistractor object when the objects appeared. These results differ from a recent study of nonhuman primate gaze following by Itakura (1996), as they provide evidence that monkeys, like great apes (Povinelli \& Eddy, 1994, 1996a, 1996b), can follow gaze cues onto specific objects.

In the first part of the trial (monkey-only condition), the stimulus monkey exhibited intense interest to a particular point in space and the subjects appear to have followed the stimulus monkey's direction of attention to this position. In terms of the definition stated in the introduction, the subjects have followed the stimulus monkey's gaze but are not joined in attention with the stimulus monkey. In the second time period (monkey-and-objects condition), a specific object appeared as a focus of the stimulus monkey's attention. The subjects looked significantly more at this target object than at the alternative distractor object. During this section of the trial, the subjects and the stimulus monkey had joint attention, as they both attended the same object (Perrett \& Emery, 1994). 
One might have expected that any salience that the target object gained from the 10 -s attention of the stimulus monkey would persist into the final part of the trial (objects-only condition), when the stimulus monkey disappeared and only the objects remained. This was not the case. The trend for the target object to be inspected more frequently that the distractor in the final period of the trials did not reach significance. Rhesus monkeys follow conspecifics' gaze to objects but may not understand the mental significance of another's attention (i.e., that the other individual is interested in the object). This would be compatible with a primitive orienting reflex (Povinelli \& Eddy, 1996a) in which monkeys follow others' gaze cues automatically.

Methodological differences may explain the discrepancies in results between this and the Itakura (1996) study. Itakura attempted to determine whether prosimians, monkeys, and apes were able to follow the gaze, head, and pointing cues of a human experimenter. Itakura required a large angle of gaze change $\left(90^{\circ}\right)$ before scoring the subject's gaze following positively. This angular change is larger than that required of the subjects in the present study. Furthermore, before providing the attention cues, the experimenter attempted to gain eye contact with each subject. Monkeys do not readily look into the eyes of humans or conspecifics (especially a more dominant individual), but apes do (Redican, 1975). Apes are more likely to look into the eyes of humans and therefore are more likely to use gaze cues. Indeed, apes may learn to use human gaze cues through socialization or enculturation, during extensive interaction with human experimenters and caregivers (Carpenter, Tomasello, \& Savage-Rumbaugh, 1995). In contrast to apes, macaque monkeys may be less willing to use the gaze of human experimenters in operant tasks because humans are perceived as more threatening. Any reluctance of macaques in this respect was circumvented in the present study by using video films of conspecifics as stimuli.

The eye region is particularly salient in primate species (Argyle \& Cook, 1976; Keating \& Keating, 1982; Perrett \& Mistlin, 1990). Baron-Cohen (1994) proposed a cognitive (and neural) module-the eye direction detector-which codes attention direction from eye gaze alone. Perrett and Emery (1994) noted that the brain mechanisms for detecting attention direction use multiple visual cues, including eye gaze, head direction, and body posture. In this study several cues to attention were oriented in the same directions. The subjects in the present study may have used eye gaze, head orientation and posture, or body posture to follow the stimulus monkey's direction of attention. The behavioral ability of monkeys to follow attention demonstrated here is consistent with the neurophysiological finding described in the introduction. Cell populations have been found that respond to direction of eyes, head, and body (Brothers \& Ring, 1993; Leonard et al., 1985; Perrett et al., 1985, 1992; Perrett \& Mistlin, 1990; Wachsmuth, Oram, \& Perrett, 1994). Such cells may contribute the appropriate neural machinery required to process the direction of another's attention from a variety of visual cues.

Primatologists have begun to test nonhuman primates' knowledge of the mental significance of attention (Ander- son, Montant, \& Schmitt, 1996; Anderson, Sallaberry, \& Barbier, 1995; Gomez, 1991; Povinelli, 1996; Whiten, in press). Studies aimed at training monkeys to use the significance of gaze cues have so far been relatively unsuccessful. Anderson et al. $(1995,1996)$ studied whether capuchin and rhesus monkeys could use human gaze and pointing cues to locate a food reward and found that the subjects could only follow manual pointing cues to the food. Similarly, Pettigrew, Forsyth, and Perrett (1993) managed to train only 2 out of 6 rhesus monkeys to follow human attention direction (defined by eye or head direction) for food reward. Thus, even though monkeys can spontaneously follow gaze of other monkeys, their ability to use gaze cues in experimental tasks appears more limited than ethological observations might suggest. Povinelli and Eddy (1996b) suggested that the ability to understand the mental significance of another's gaze is a dissociable ability from simple gaze following, although one may be a precursor to the other (Baron-Cohen, 1994; Perrett \& Emery, 1994). Whiten (1996) speculated that behavior reading, or inferring goaldirected behavior, emotion, and intention from external perceptual signals and a representation of an individual's behavioral patterns, may have developed through evolution into a mind-reading ability or a theory-of-mind mechanism (Premack \& Woodruff, 1978).

\section{References}

Anderson, J. R., Montant, M., \& Schmitt, D. (1996). Rhesus monkeys fail to use gaze direction as an experimenter-given cue in an object-choice task. Behavioral Processes, 37, 47-55.

Anderson, J. R., Sallaberry, P., \& Barbier, H. (1995). Use of experimenter-given cues during object-choice tasks by capuchin monkeys. Animal Behavior, 49, 201-208.

Altmann, S. A. (1967). The structure of social communication. In S. A. Altmann (Ed.), Social communication among primates (pp. 325-362). Chicago: University of Chicago Press.

Argyle, M., \& Cook, M. (1976). Gaze and mutual gaze. Cambridge, UK: Cambridge University Press.

Baron-Cohen, S. (1994). How to build a baby that can read minds: Cognitive mechanisms in mindreading. Current Psychology of Cognition, 13, 513-552.

Brothers, L. (1990). The social brain: A project for integrating primate behavior and neurophysiology in a new domain. Concepts in Neuroscience, 1, 27-51.

Brothers, L., \& Ring, B. (1993). Medial temporal neurons in the macaque monkey with responses selective for aspects of social stimuli. Behavioral Brain Research, 57, 53-61.

Bruce, C., Desimone, R., \& Gross, C. G. (1981). Visual properties of neurons in a polysensory area in superior temporal sulcus of the macaque. Journal of Neurophysiology, 46, 369-384.

Butterworth, G. (1991). The ontogeny and phylogeny of joint visual attention. In A. Whiten (Ed.), Natural theories of mind: Evolution, development and simulation of everyday mindreading (pp. 223-232). Oxford, UK: Blackwell.

Butterworth, G., \& Cochran, E. (1980). Towards a mechanism of joint visual attention in human infancy. International Journal of Behavioral Development, 3, 253-272.

Butterworth, G., \& Jarrett, N. (1991). What minds have in common is space: Spatial mechanisms serving joint visual attention in infancy. British Journal of Developmental Psychology, 9, 55-72.

Carpenter, M., Tomasello, M., \& Savage-Rumbaugh, S. (1995). 
Joint attention and imitative learning in children, chimpanzees and encultured chimpanzees. Social Development, 4, 217-237.

Chance, M. R. A. (1967). Attention structure as a basis of primate rank orders. Man, 2, 503-518.

Corkum, V., \& Moore, C. (1994). Development of joint visual attention in infants. In C. Moore \& P. Dunham (Eds.), Joint attention: Its origin and role in development (pp. 61-85). Hillsdale, NJ: Erlbaum.

Gomez, J. C. (1991). Visual behavior as a window for reading the mind of others in primates. In A. Whiten (Ed.), Natural theories of mind (pp. 195-208). Oxford, UK: Blackwell.

Itakura, S. (1996). An exploratory study of gaze-monitoring in nonhuman primates. Japanese Psychological Research, 38, 174-180.

Keating, C. F., \& Keating, E. G. (1982). Visual scan patterns of rhesus monkeys viewing faces. Perception, 11, 211-219.

Leonard, C. M., Rolls, E. T., Wilson, F. A. W., \& Baylis, G. C. (1985). Neurons in the amygdala of the monkey with responses selective for faces. Behavioral Brain Research, 15, 159-176.

Menzel, E. W., \& Halperin, S. (1975). Purposive behavior as a basis for objective communication between chimpanzees. Science, $189,652-654$.

Mineka, S., Davidson, M., Cook, M., \& Keir, R. (1984). Observational conditioning of snake fear in rhesus monkeys. Journal of Abnormal Psychology, 93, 355-372.

Perrett, D. I., \& Emery, N. J. (1994). Understanding the intentions of others from visual signals: Neurophysiological evidence. Current Psychology of Cognition, 13, 683-694.

Perrett, D. I., Hietanen, J. K., Oram, M. W., \& Benson, P. J. (1992). Organization and functions of cells responsive to faces in the temporal cortex. Philosophical Transactions of the Royal Society of London: Biological Sciences, 335, 23-30.

Perrett, D. I., \& Mistlin, A. J. (1990). Perception of facial characteristics by monkeys. In W. C. Stebbins \& M. A. Berkley (Eds.), Comparative perception (Vol. II, pp. 187-215). New York: Wiley.

Perrett, D. I., Rolls, E. T., \& Caan, W. (1982). Visual neurons responsive to faces in the monkey temporal cortex. Experimental Brain Research, 47, 329-342.

Perrett, D. I., Smith, P. A. J., Potter, D. D., Mistlin, A. J., Head, A. S., Milner, A. D., \& Jeeves, M. A. (1985). Visual cells in the temporal cortex sensitive to face view and gaze direction. Proceedings of the Royal Society of London B, 223, 293-317.

Pettigrew, L., Forsyth, H., \& Perrett, D. I. (1993). Training rhesus monkeys to use human head and gaze direction in a visual discrimination task. Unpublished manuscript.

Povinelli, D. J. (1996). Chimpanzee theory of mind?: The long road to strong inference. In P. Carruthers \& P. K. Smith (Eds.),
Theories of theories of mind (pp. 293-329). Cambridge, UK: Cambridge University Press.

Povinelli, D. J., \& Eddy, T. J. (1994). The eyes as a window: What young chimpanzees see on the other side. Current Psychology of Cognition, 13, 695-705.

Povinelli, D. J., \& Eddy, T. J. (1996a). Chimpanzees: Joint visual attention. Psychological Science, 7, 129-135.

Povinelli, D. J., \& Eddy, T. J. (1996b). What young chimpanzees know about seeing. Monographs of the Society for Research in Child Development, 61(3, Serial No. 247).

Premack, D., \& Woodruff, G. (1978). Does the chimpanzee have a theory of mind? Behavioral and Brain Sciences, 4, 515-526.

Redican, W. K. (1975). Facial expressions in nonhuman primates. In L. A. Rosenblum (Ed.), Primate behavior: Developments in field and laboratory research (Vol. 4, pp. 103-194). New York: Academic Press.

Scaife, M., \& Bruner, J. S. (1975). The capacity for joint visual attention in the infant. Nature, 253, 265-266.

van Schaik, C. P., van Noordwijk, M. A., Warsono, B., \& Sutriono, E. (1983). Party size and early detection of predators in Sumatran forest primates. Primates, 24, 211-221.

Wachsmuth, E., Oram, M. W., \& Perrett, D. I. (1994). Recognition of objects and their component parts: Responses of single units in the temporal cortex of the macaque. Cerebral Cortex, 4 , 509-522.

Whiten, A. (1996). When does smart behavior-reading become mind-reading? In P. Carruthers \& P. K. Smith (Eds.), Theories of theories of mind (pp. 277-292). Cambridge, UK: Cambridge University Press.

Whiten, A. (in press). Evolutionary and developmental origins of the mindreading system. In J. Langer \& M. Killen (Eds.), Piaget, evolution and development. London, UK: Erlbaum.

Whiten, A., \& Byrne, R. W. (1988). The manipulation of attention in primate tactical deception. In R. W. Byrne \& A. Whiten (Eds.), Machiavellian intelligence: Social expertise and the evolution of intellect in monkeys, apes and humans (pp. 211-223). Oxford, UK: Oxford University Press.

Whiten, A., \& Ham, R. (1992). On the nature and evolution of imitation in the animal kingdom: Reappraisal of a century of research. In P. J. B. Slater, J. S. Rosenblatt, C. Beer, \& M. Milinski (Eds.), Advances in the study of behavior (pp. 239283). San Diego, CA: Academic Press.

Received September 25, 1996

Revision received November 28, 1997 Accepted January 30, 1997 . 